\title{
Le paysage de l'investissement durable
}

Écrit par : Monika Dutt, Senior Investment Specialist at Vanguard

Dernière mise à jour : 17 août 2020

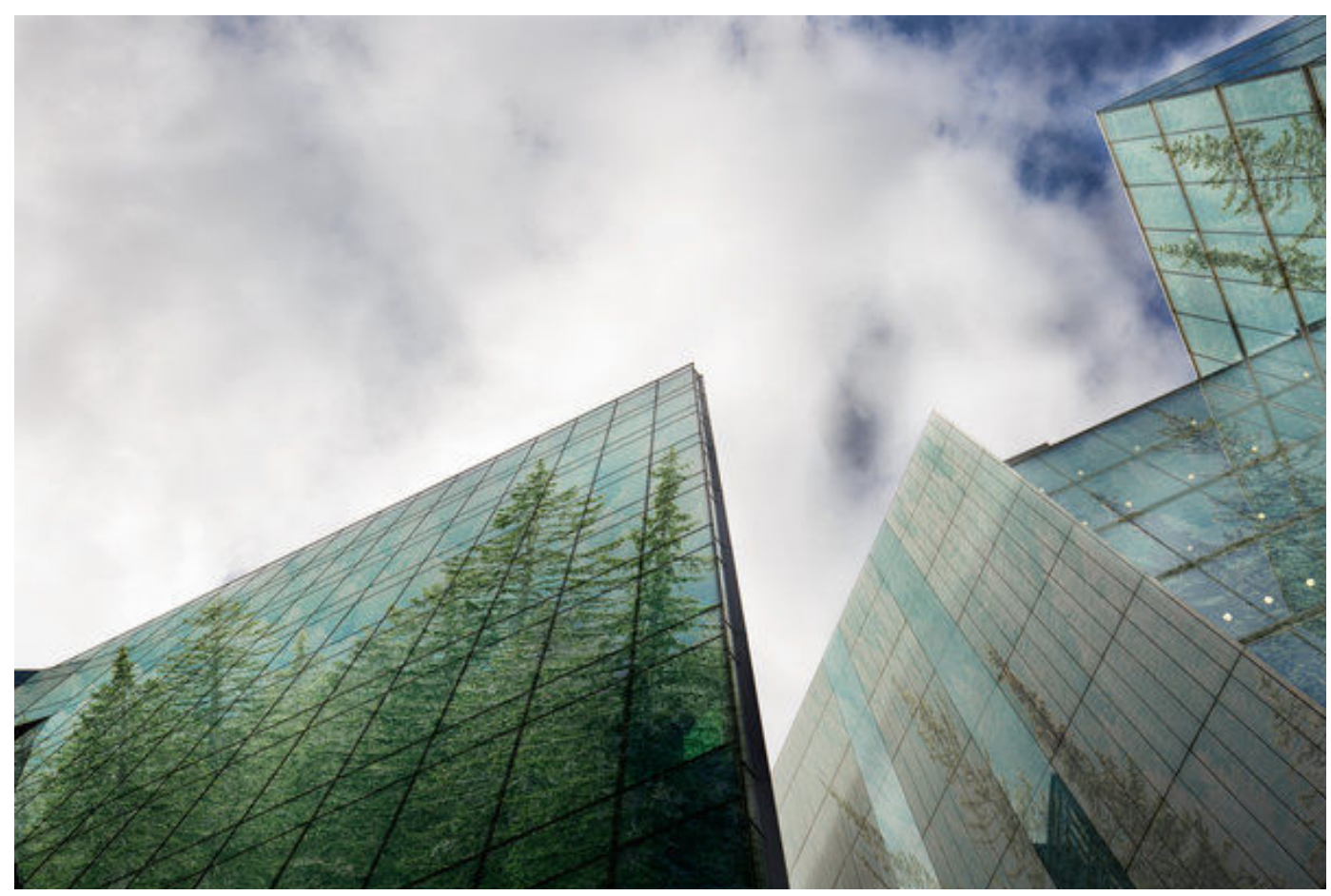

Le paysage de l'investissement durable

Dans un paysage mondial en mutation, les stratégies

d'investissement durable ont le vent en poupe. Début 2018 , les actifs durables ont atteint 30.7 billions US $\$$ au niveau mondial. Ventilés par région, la plus grande partie de ces actifs étaient gérés en Europe (46\%), suivie des États-Unis (39\%), du Japon et du Canada (6-7 \% chacun).

Depuis 2016, l'investissement durable a progressé de $34 \%$ dans le monde. Cette croissance remarquable peut s'expliquer par un ensemble de facteurs complexes, notamment la meilleure disponibilité de données sur l'investissement durable et l'augmentation des initiatives en faveur de l'investissement responsable.

À l'heure actuelle, plus de 11700 entreprises faisant appel à l'épargne publique ont communiqué des données relatives à l'investissement durable, mesurées sur 
la base d'indicateurs environnementaux, sociaux et de gouvernance (ESG). En outre, plus 125 organisations, telles que FTSE, MSCI et Sustainalytics, mènent des recherches sur les critères ESG et établissent des notations. Dans l'ensemble, un accès plus aisé aux données ESG a permis d'intégrer des filtres de durabilité dans le processus d'investissement.

De plus en plus d'initiatives en faveur de l'investissement responsable, y compris l'Accord de Paris sur le climat et le Programme de développement durable à l'horizon 2030 des Nations Unies, contribuent à orienter les actifs vers des stratégies privilégiant les critères ESG. En outre, les responsables publics partout dans le monde prennent des dispositions réglementaires visant à définir et promouvoir l'investissement durable.

La proposition la plus complète à ce jour émane de l'Union européenne. La Commission européenne a dévoilé un plan d'action sur la finance durable ayant pour but d'établir un système de classification clair des activités durables, de définir les responsabilités qui incombent aux gestionnaires d'actifs et aux investisseurs institutionnels et d'intégrer les préoccupations climatiques dans les processus de gestion des risques des banques.

Aux États-Unis, l'approche réglementaire de l'investissement durable est moins prescriptive. En 2015, le ministère du Travail a publié des orientations sur les critères ESG, qui reconnaissent que ces critères peuvent induire des risques significatifs pour les entreprises. Aussi, le ministère autorise la mention des critères ESG dans les déclarations de principes relatives aux investissements, mais sans la rendre obligatoire.

Les initiatives en faveur de la finance durable se développent aussi en Asie. En Chine, les entreprises cotées seront tenues de faire connaître leurs risques ESG à partir de 2020. De même, la bourse de Hong Kong envisage de rendre obligatoire la publication d'informations ESG pour les sociétés faisant appel à l'épargne publique.

Alors que les données sur l'investissement durable se multiplient et que différentes approches réglementaires sont mises en place à l'échelle internationale, la terminologie ESG va probablement devenir de plus en plus difficile à comprendre. Pour y voir plus clair, nous classons les stratégies d'investissement durable en quatre catégories, à savoir l'intégration des critères ESG, le filtrage de portefeuille, l'actionnariat actif et l'investissement à impact positif.

As sustainability data continues to proliferate and different international regulatory approaches keep being introduced, ESG vocabulary will likely become even more difficult to understand. To help clarify the confusion, we categorise sustainable investing strategies into four categories, namely ESG integration, portfolio screening, active ownership and impact investing. 


\section{Stratégies d'investissement durable}

De façon générale, les investisseurs qui décident d'intégrer des critères ESG ont pour but d'améliorer le profil de risque et de rendement de leurs portefeuilles. À titre d'exemple, ils peuvent sous-pondérer ou écarter les entreprises qui présentent des risques ESG élevés, car ces risques peuvent se traduire par des pertes financières. Les partisans de cette approche ne sont pas forcément animés par des valeurs sociales ou environnementales. Ils se contentent d'étudier les données ESG en vue de rationaliser leurs processus d'investissement existants.

L'approche fondée sur le filtrage de portefeuille consiste à sélectionner les entreprises en fonction de critères spécifiques. Cette méthode repose sur un filtrage positif ou négatif. Un filtrage négatif sous-pondère ou exclut les entreprises du portefeuille si elles ne répondent pas aux critères ESG attendus par les investisseurs. Par exemple, un portefeuille qui exclut les entreprises des secteurs du pétrole et du gaz, comme Royal Dutch Shell et Exxon Mobil, suit cette approche.

Par comparaison, un filtrage positif surpondère ou sélectionne uniquement les entreprises qui affichent une notation ESG plus élevée que la moyenne du secteur. Un portefeuille qui comprend uniquement des entreprises qui respectent les principes du Pacte mondial des Nations Unies correspond à cette méthode.

L'actionnariat actif est une autre stratégie d'investissement durable. Dans ce cas de figure, les créanciers ou les actionnaires peuvent exercer une influence sur les pratiques de gestion des entreprises bénéficiaires des investissements en participant aux actions de sensibilisation sectorielles, aux initiatives d'implication des salariés et au vote par procuration.

Les stratégies d'investissement à impact positif poursuivent généralement deux objectifs : améliorer les résultats financiers et avoir un effet bénéfique sur la société ou sur l'environnement. Les obligations à impact social qui financent des programmes reposant sur le principe du paiement aux résultats sont une illustration de cette approche.

Performance : l'équilibre entre exposition aux critères ESG et rendement boursier

Les investisseurs en quête de stratégies durables doivent arbitrer entre exposition aux critères ESG et rendement sur le marché, mesuré par le niveau des indices pondérés par capitalisation boursière, tels que le S\&P 500, le FTSE 100 et le CAC 40.

Intuitivement, cette approche est sensée. Une stratégie qui applique des filtres ESG à un large éventail de titres offrira probablement un profil de rendement différent d'un indice de référence. Une façon d'évaluer cette différence consiste à calculer l'erreur de suivi (tracking error), parfois appelée risque actif. L'erreur de suivi décrit la façon dont un fonds s'éloigne de la composition de son indice de 
référence sur une certaine période, et une erreur de suivi élevée indique que le risque actif est plus important.

CL'Observateur de l'OCDE Novembre 2019

\section{Références}

Global Sustainable Investment Alliance (2018), 2018 Global Sustainable Investment Review http://www.gsi-alliance.org/wp-content/uploads/2019/03/ GSIR_Review2018.3.28.pdf

Grim, D. M. and D.B. Berkowitz (2018), ESG, SRI, and impact investing: A primer for decision-making, https://personal.vanguard.com/pdf/ISGESG.pdf

European Commission (2019), Green finance https://ec.europa.eu/info/businesseconomy-euro/banking-and-finance/sustainable-finance_en

Le paysage de l'investissement durable http://dx.doi.org/https://www.dol.gov/ agencies/ebsa/employers-and-advisers/guidance/field-assistance-bulletins/ 2018-01

HKEX (2019), Review of the environmental, social and governance reporting guide and related listing rules https://www.hkex.com.hk/-/media/HKEXMarket/News/Market-Consultations/2016-Present/May-2019-Review-of-ESGGuide/Consultation-Paper/cp201905.pdf?la=en 